\title{
TAB-PRRA: Improved Link Repair Decision Mechanism for On- demand Routing in MANET
}

\author{
Ms. Deepika Patil, Mrs. Nitika Vats Doohan \\ Research Scholar, Dept. of CSE SDBCT, Indore (M.P), India \\ Associate Prof., Dept. of CSE SDBCT, Indore (M.P), India
}

\begin{abstract}
The traditional AODV protocol uses a fixed hop count based threshold as a basis for deciding when to invoke Local Repair or Source Repair in case of a link break. The problem arises with this type of repairing mechanism is that the network is not clear that at which time what algorithms needs to be applied. So some condition has to be identified on the basis of which the decision can be taken. Also the decision must be preemptive so that the loss of data can be avoided before occurrence. In this paper, a Threshold Alarm Based Preemptive Route Repair Algorithm (TAB-PRRA) for Multi-Hop Local repair in ADOV is presented and simulated. It focuses on measuring those parameters which provides an prior information of link failure before its occurrence and also if the link break occurs that the network must know the time to start the route discovery. The approach offers efficient utilization of network resources such as energy and bandwidth.

Index Terms: MANET, AODV, TAB (Threshold Alarm Based), PRRA (Pre-emptive Route Repair Algorithm), RREQ, RREP, Local Repair;
\end{abstract}

\section{INTRODUCTION}

Mobile ad hoc network (MANET) is the most innovative and challenging area of wireless networking. MANET is an autonomous collection of mobile users that communicate over relatively band width constrained wireless

links. Some situations in which user-required infrastructure is not available, cannot be installed, or cannot be installed in time in a given geographic area. In these situations, mobile ad hoc network provides the needed connectivity and network services.

Routing in MANET is a Dynamic Optimization Problem as the search space changes over time. The routing policy is defined as the rule that specifies what node to take next at each decision node to reach the destination node. Due to the time varying nature of the topology of the networks, traditional routing techniques such as distance-vector and link-state algorithms that are used in fixed networks, cannot be directly applied to mobile ad hoc networks. The constraints of MANETs demand the need of specialized routing algorithms that can work in a decentralized and self-organizing way. The routing protocol of a MANET must dynamically adapt to the variations in the network topology.

The routing scheme in a MANET can be classified into two major categories - Proactive and Reactive [1]. The proactive or table driven routing protocols maintain routes between all node pairs all the time. It uses periodic broadcast advertisements to keep routing table up-to-date. This approach suffers from problems like increased overhead, reduced scalability and lack of flexibility to respond to dynamic changes. The reactive or on-demand approach is event driven and the routing information is exchanged only when the demand arises. The routing is initiated by the source.

\section{RELATED STUDY}

In 2005 Hui-Yao An, Ling Zhong, Xi-Cheng Lu, and Wei Peng proposed A Cluster-Based Multipath Dynamic Source Routing in MANET (CMDSR) that is designed to be adaptive according to network dynamics [12]. It uses the hierarchy to perform Route Discovery and distributes traffic among diverse multiple paths. The CMDSR is based on a 2-level hierarchical scheme: the 1-cell cluster and 2-server cluster. The main idea of this proposition is to transfer the Route Discovery procedure to the 2-server level to prevent the network flooding due to the DSR Route Discovery. Thus, Route Discovery does not require flooding mechanism and overhead is minimized and improve the networks scalability. The route overhead increases significantly with an increasing node density and a significant number of nodes in the plane structure, so the scalability is hard.

In 2010 Zhiming $\mathrm{Xu}$, Yu Wang and Jingguo Zhu proposes an approach based on the ODMRP (OnDemand Multicast Routing Protocol) in MANET (Mobile Ad hoc Network), a reliable ODMRP (R-ODMRP) is proposed for preferable throughput and especially suited for high-speed MANET, which includes packet acknowledgement, lost packet recovery, secure authentication and QoS based packet delivery [10]. With the exploration of active network, R-ODMRP constructs the multicast routing based on the cluster, establishes a distributed mechanism of the acknowledgment 
and recovery of packet delivery. Along with cluster key distributed in one cluster, this protocol can authenticate the consistency of multicast source and receivers depending on local security strategy. The specific mesh links are adaptively chosen by virtue of the descriptive QoS vectors meanwhile, the forwarding nodes can flexibly schedule different multicast packets according to the types of the multicast applications. The performance of the proposed schemes is evaluated based on the network simulator and achieves a significant improvement.

In 2011 Jyoti Jain, Roopam Gupta and T.K. Bandhopadhyay gives a general survey of research on local repair of link, if it is broken during communication for MANET and proposes a new local repair scheme in order to make up the deficiency of the existing local repair schemes. The improved local repair scheme concerns about the over head requirement and end to end delay in transmission. Nodes are required to keep the next twohop node address for each route entry in routing table. During local repair, the repairing node use Ant algorithm for finding new route for next to next node in the link considering that other part of the link is already in existence. Reduced size of F-ANT and B-ANT will give significant reduction in overhead [4]. In this case repairing node not only tries to discover the route to the destination node of data packet, but also attempts to establish the route to its downstream node (i.e. the next hop node). The proposed algorithm will be highly adaptive, scalable and efficient and mainly reduces end-to-end delay in high mobility cases.

In 2003 Srdjan Krco and Marina Dupcinov proposed Improved Neighbor Detection Algorithm for AODV Routing Protocol for the improvement of link quality [7]. This algorithm is developed to check the quality of link as a function of SNR. Link with better quality factor are selected for the communication, In this algorithm only good neighbors are kept in routing tables all routes are established over good quality links. This ensures that both broadcast and unicast messages have equal chances of being received correctly regardless of the used transmission rate.

Ionut D. Aron et al [8] proposed a localized route repair (LRR) technique, which repairs a route on the fly as soon as it is broken and eliminates the need for network-wide flooding. LRR technique resulted in an enormous reduction in overhead when simulated on an event driven packet level simulator and results in nearly 30 percent overhead savings. This technique improves other network characteristics as well.

Sergio Crisstomo, et. al. proposes an extension to the AODV protocol, denoted Preemptive Local Route Repair (PLRR) [7], that aims to avoid route failures by preemptively local repairing routes when a link break is about to occur. This protocol is to enhance node's information concerning link stability to its neighbors resorting to HELLO messages. These messages are appended with a mobility extension containing the node's position, motion vector and an associated timestamp. This mobility information will be used to predict the instant $a$ link between two neighbors will break. This proposal does not take into account the sender and destination location information, as other location aided routing protocols. This proposal aims at improving the AODV Quality of Service capabilities by minimizing route failure.

In 2011 S R. Azzuhri, M Portmann, W L Tan proposes a novel decision based method for link repairing \& identification of repairing algorithm. According to this AODV uses a fixed Local Repair threshold in terms of number of hops from the destination where a link break occurs, to determine if to invoke Source Repair or Local Repair. This Paper define a Local Repair Threshold parameter that determines how far along the end-to-end path that a link break needs to occur in order to initiate Local Repair, as opposed to Source Repair [9]. The simulation results show that the optimal choice of the Local Repair Threshold, in terms of Packet Delivery Ratio depends on the network load.

\section{Problem Identification}

Ad hoc Networks are wireless networks without any fixed infrastructure. The network topology changes frequently and unpredictably due to the random movement of the nodes. The Ad Hoc on Demand Distant Vector Routing (AODV) protocol works in a dynamic fashion, by establishing a route on demand and continues that route until it breaks [11]. This movement of nodes \& heavy traffic of data packets the link failure occurs. In existing AODV protocol the detection of this unpredictable nature of node is not identified. Along with that decision of selecting the repairing mechanism is not clarified. While considering the repairing mechanism the link failure gives a triggering of new route discovery mechanism. Thus we required a preemptive approach which is been able to detect the link failure before its occurrence [15]. This is done by the link parameters like node energy, link strength, packet delivery ratio etc. Even if the link break occurs than this work will make an active decision of how the algorithm is been selected accordingly.

- To Provides Loop Free Route Discovery

- To Provide Reputation Free Routes

- To Provide Preemptive Route Failure Detection.

- To identify the link Break Condition

- To better take the decision of which route repair mechanism

- To Provide an break free routing which saves cost, Time \& Energy 
Link Decision Parameters:-The predictive approach will shows that a flexible, parameterized and adaptive approach to choosing the Local Repair Threshold, can improve the Packet Delivery Ratio by up to 37\% (in absolute terms), compared to the approach employed by standard AODV [13]. It also gives a clear description for using such a fixed threshold as a basis for the decision making is suboptimal, since the optimal choice of the threshold depends on the level of network load. This work have demonstrated that an optimal choice of the threshold TLR can result in a significant performance improvement, compared to both the fixed threshold used in AODV, and the Source Repair only approach taken by DYMO [15].

This paper makes a use of a more flexible, parameterized and adaptive choice of route repair strategies than is implemented by current MANET and Wireless Mesh routing protocols such as AODV.

Identified Issue:- One of the key open research questions, which these papers have not addressed, is how to determine the optimal Local Repair Threshold TLR for different network scenarios and different levels of network load. This is the main focus of our proposed TAB-PRRA having multi-hop link failure identification for Local repair in AODV.

Characteristics of ad hoc networks include resource-poor devices, limited bandwidth, high error rates, and a continually changing topology. Among the available resources, battery power is typically the most constraining. Hence, routing protocol must have Minimal control overhead, Minimal processing overhead, Multi-hop routing capability, Dynamic topology maintenance and Loop prevention. Many proactive and reactive routing are already in existence [17]. The routing tables of the nodes within the neighborhood are organized to optimize response time to local movements and provide quick response time for requests for establishment of new routes. Primary objectives of this algorithm are [18]:

$>$ To broadcast discovery packets only when necessary [14].

$>$ To distinguish between local connectivity management, neighborhood detection and general topology maintenance.

$>$ To disseminate information about changes in local connectivity to those neighbouring mobile nodes that is likely to need the information.

\section{PROPOSED TAB-PRRA APPROACH}

In this proposed TAB-PRRA mechanism a routing path is constructed when a node needs to communicate with other network in its specific range. Communication starts with route discovery for data transmission from source to destination. If route exists then data is directly sent otherwise a new route request (RREQ) is been flooded in the network. Then the destination node replies with a route reply packet (RREP). After successful retrieval of route reply new route can be formed. In some cases few of the link is been disconnected or broken due to environmental factors. Due to that data loss occurs. Thus the link needs to be verified before the data transmission. If the link break occurs then the decision must be taken at proper time to initiate source repair or local repair mechanism.

For achieving the above mentioned goals this work focuses on a novel Threshold Alarm based preemptive route repair algorithm (TAB-PRRA). In this initially a source node send the Hello packet to measure the current status on the link \& nodes. This Hello packet contains Battery Life, TTL Value \& Node mobility field o the basis of which some average threshold value is calculated. After this rank is been given to each links representing their strength. During the data transmission if the value of threshold for this mentioned link is below than the defined average threshold then new route discovery is initiated giving the pre-emptive signal of future link break, but the route is still continue. Again if the value of this strength is lower than this min (min (threshold)) than route will be changed and an alarm message is been issued to each node so that updating can be done in routing table. After this pre-emptive approach decision is taken to initiate the link repair either source or local repair. This decision is based on the network value in distance covered by transmitted data packet. If the failure occurs at neighbor nodes of source than the source repair is initiated otherwise the local repair can work smoothly. The proposed algorithm is mentioned below:-

\section{Threshold Alarm Based Preemptive Route Repair Algorithm (TAB-PRRA)}

The complete scenario for the proposed approach is executed in 3 step algorithms. These are:-

\section{Shortest Path Algorithm}

1. Start with the Node Creation for First User.

2. Created nodes are classified by Co-ordinates $(\mathrm{X}, \mathrm{Y})$.

3. After this the scenario is generated for communication

4. Every node has a pre-defined range, in which it can detect the presence of other nodes. The following strategy is adopted when a node needs to find a path to an unknown destination.

5. Identification of Nodes transmission range by 4 Quadrant Clock techniques. 
Fig. 1. Check the range in Positive Quadrant Clockwise Direction

Fig. 2. Check the range in Negative Quadrant Clockwise Direction

Fig. 3. Check the range in Down Positive Quadrant Clockwise Direction

Fig. 4. Check the range in Down Negative Quadrant Clockwise Direction

6. This approach is particularly useful in determining the shortest path (minimum number of hops) to the destination.

\section{Transmission of Data Form Source to Destination Algorithm}

1. Next, the user requests for the transmission of data from the source to the destination.

2. Node $\mathrm{S}$ wants to communicate with $\mathrm{D}$

3. Initiate route identification mechanism

a. If $\mathrm{D}$ is a neighbor of $\mathrm{S}$

b. Route available for data transmission

4. Otherwise $\mathrm{S}$ Checks its routing table for its neighbor.

5. If no route is present

6. Start new route discovery

7. Source $\mathrm{S}$ sends new RREQ to its entire neighbor.

8. This message is flooded till $\mathrm{D}$ receives this.

9. After this D reply with RREP.

10. Communication Starts.

Preemptive Route Repair Algorithm (TAB-PRRA)

1. Each node measures the link strength after every $10 \mathrm{sec}$.

2. This value gives the threshold on the basis of following parameters

3. Threshold (Battery power, Packet delivery Ratio, TTL Value, No of Packet Relayed, Node Mobility within the range)

Threshold $=[$ Initial Battery Power $+($ Packet deliver in Unit Time/ Total number of Packet $)+$ Total Time Taken by Packet +Total number of packet relayed+ Mobile Nodes in a network] * $(40 / 100)$

2. We then investigate what the optimal choice is of this threshold for a range of network scenarios. We define the link break location parameter $\mathbf{l}_{\mathbf{l b}}$ as follows:

$$
1_{\mathrm{lb}}=\text { (hop index of broken link / Total number of hops in Path) }
$$

3. Each node shares this information \& gives rank to every other node \& link.

4. If the links condition $<$ min threshold

4. Initiate new route discovery

5. End If threshold $<\min$ (min threshold)

5. Issue Route Change Alarm message to source

6. Each node shares this message \& updates their routing table.

\section{COMParison AnAlysis}

The approach is simulated and its results are analyzed and compared with existing approach on the basis of certain network performance parameters such as packet delivery rate, throughput, routing load etc. Packet delivery rate is measured as ratio between the received packets and send packet. Throughput is the rate of data bits sent by nodes per time units. Routing load is the total no of control packets flooded in the network. The results are analyzed and shown using below graphs. In this the simulated approach improves network performance and make able to take right decision for route repair. Result compared with existing approach considering all above parameters. 


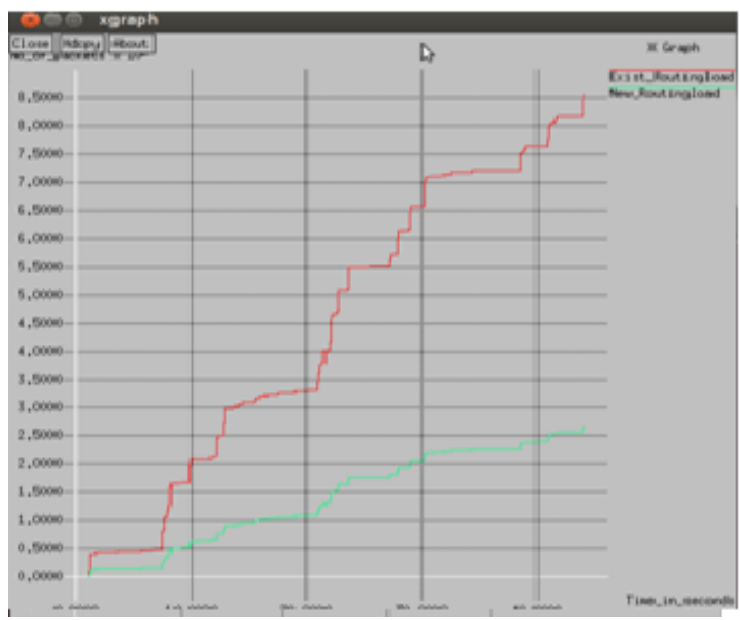

Figure 1: Packet Delivery Rate Comparisons

From the above results it is proved that the proposed mechanism is providing better and accurate approach for timely decision making. It also gives the effective results in case of all the three working parameters. Red line is measuring the existing AODV link repair while green line is used to show the proposd TAB-PRRA approach results. It gives the benefitted output. Throughput and packet delivery is high for the proposed approach while for existing it is very low. Also in existing meachnims each time the local or source repair decision has to taken but in this work the decision is always be local repair after the $50 \%$ network is crossed out by the packets.

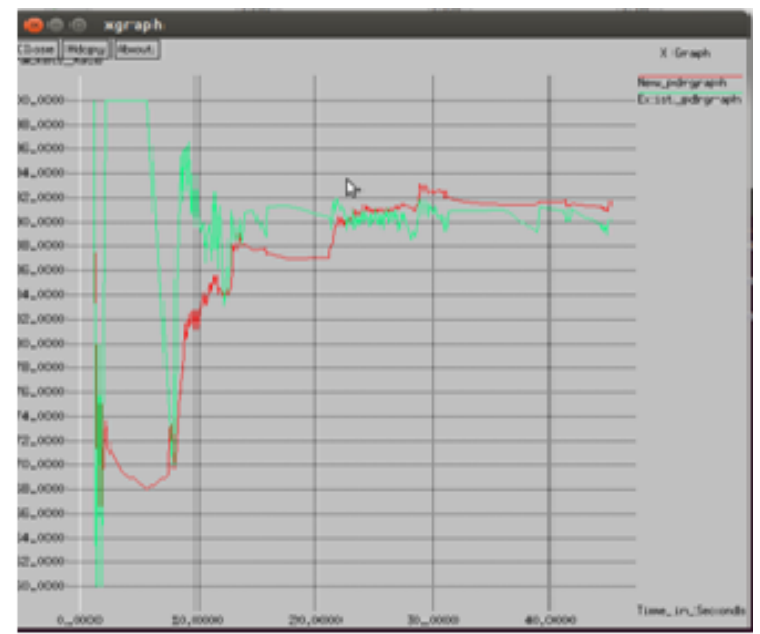

Figure 2: Throughput Comparison

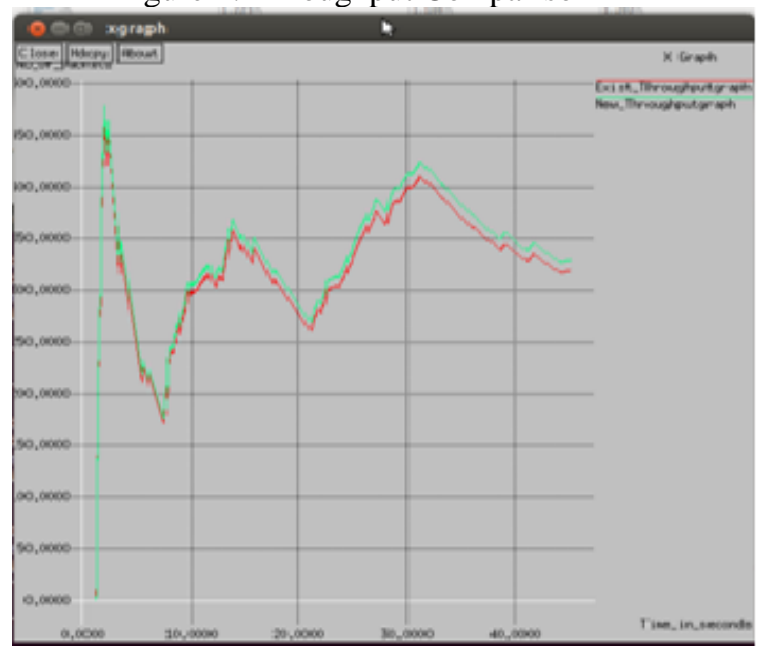

Figure 3: Routing load Comparison 
Thus to make the effective and accurate decision for which link repair had to be intialised and must sends an update packets or an alrm packets to intimate the other node about the detected failures. It also saves the energy and efforts. The network lifetime is also increased by using the proposed approach. Thus at the intial level of our work the approach s providing efficient results to direct the future outcomes.

\section{CONCLUSION}

In MANET, the descision of selecting route repair mechansim is main concern. The performance of network is based on the decsison. The simulated approach will shows an improved performance and maximize network liftim, data transmission at different nodes. The alternative route construction process could be initiated at any time, not just when a route has failed. The dynamically constructed alternative route's information is passed on to the upstream nodes, which then determine by themselves when to direct their packets to the "optimal" alternative route. Thus by applying the threshold based approach and an alarm generation at early stages of link failure will definitely provide the better result in future

\section{FUTURE WORK}

As a future work, improvement in the proposed scheme can be accomplished in terms of both control traffic with minimum data loss incurred and resulting gain in path optimality by our proposed TAB-PRRA. Indeed, the proposed scheme detects the best links based on its strength, if any, only between the nodes which are located 2-hop away to each other. Therefore, as a next step, we would like to develop a route repairing \& optimization scheme which is able to detect prior link failures between any pair of nodes on a connection. Finally, such a fault tolerance route repair scheme can lead for effective transmission without worrying about the path optimality \& link condition, which has been addressed as a problem in local recovery operations.

\section{ACKNOWLEDGMENT}

The authors wish to acknowledge SDBCT administration for their support \& motivation during this research. The authors would also like to thank the anonymous referees for their many helpful comments, which have strengthened the paper. They also like to give thanks to Mrs. Nitika Vats Doohan for discussion regarding the cloud security policies \& for producing the approach adapted for this paper.

\section{REFERENCES}

[1] Saaidal R. Azzuhri, Marius Portmann \& Wee Lum Tan , "Evaluation of Parameterised Route Repair in AODV" in IEEE Transaction 2011.

[2] Zeki Bilgin \& Bilal Khan "A Dynamic Route Optimization Mechanism for AODV in MANETs “ in IEEE Transaction 2010.

[3] S. Revathi \& T. R. Rangaswamy , "Secured Optimal Adaptable Ad-Hoc Routing Protocol in MANET S". in European Journal of Scientific Research Issn 1450-216, Vol.73 No.1, 2012.

[4] Jyoti Jain, Roopam Gupta in T.K. Bandhopadhyay, "On Demand Local Link Repair Algorithm for AODV Protocol “ in IJCA Volume 35- No.5, December 2011.

[5] Rachid Haboub \& Mohammed Ouzzif, “Secure \& reliable routing in MANET” in IJCSES Vol.3, No.1, February 2012.

[6] Nadia Qasim, Fatin Said \& Hamid Aghvami, "Mobile Ad Hoc Networking Protocols' Evaluation through Simulation for Quality of Service" in IAENG, 36:1, IJCS 36_1_10,February 2009.

[7] P. Priya Naidu1 \& Meenu Chawla, "Extended Ad Hoc on Demand Distance Vector Local Repair Trial for MANET" in IJWMN, Vol. 4, No. 2, April 2012.

[8] K.Muthumayil, V.Rajamani \& S.Manikandan, “Ad-Hoc Cross Layered Energy based on-demand Routing Protocol for MANETs” in Conference in R.M.D Engg.College, Gummudipoondi, Dec-2011.

[9] Fikret Sivrikaya, Sahin Albayrak \& Bulent Yener, "Spatially Limited Contention for Multi-Hop Wireless Networks" in IEEE Globecom Issn 978-1-4244-2324-8/08 IEEE. 2008.

[10] Zhiming Xu ,Yu Wang \& Jingguo Zhu, "A Reliable Multicast Routing Protocol for High-speed Mobile Ad Hoc Networks: RODMRP" in Journal of Software, Vol 9, No 2, 2009.

[11] S. Subburam and P. Sheik Abdul Khader, "Efficient Two Hop Local Route Repair Mechanism Using Qos-Aware Routing for Mobile Ad Hoc Networks" in Indian Journal of Science and Technology ISSN:0974-6846 Vol:5 Issue:11 November 2012

[12] Hui-Yao An, Ling Zhong, Xi-Cheng Lu, and Wei Peng, "A Cluster-Based Multipath Dynamic Source Routing in MANET" in IEEE Transaction, 2005.

[13] S. Revathi \& T. R. Rangaswamy , "Dynamic Route Shortening and Route Repairing Mechanism for Mobile Ad Hoc Networks" in Journal of Computer Science ISSN 1549-3636 8 (8): 1212-1218, 2012.

[14] Jianlin Guo, Chuan Han, Philip Orlik \& Jinyun Zhang, "Loop-Free Routing in Low-Power and Lossy Networks" in Sensorcomm IARIA, ISBN: 978-1-61208-207-3, 2012.

[15] J.-N. LIU and Imrich Chlamtac "Mobile ad hoc networking with a view of 4G wireless: Imperatives and Challenges".

[16] C. S. R. Murthy and B. Manoj "Ad Hoc Wireless Networks: Architectures and Protocols", Prentice Hall PTR, May 2004.

[17] Elizabeth.M.Royer, C-K Toh," A Review of Current Routing Protocols for Ad-hoc Mobile Wireless networks",IEEE personal communication,pp.46-55,April 1999.

[18] C. E. Perkins, E. M. Royer, and S. R. Das, “Ad hoc on-demand distance vector routing,” IETF RFC 3561 , July 2003. 